\title{
Barriers and opportunities for refugee mental health services: clinician recommendations from Jordan
}

\section{Policy and Systems Brief Report}

Cite this article: Al-Soleiti M, Abu Adi M, Nashwan A, Rafla-Yuan E (2021). Barriers and opportunities for refugee mental health services: clinician recommendations from Jordan. Global Mental Health 8, e38, 1-6. https://doi.org/10.1017/gmh.2021.36

Received: 8 February 2021

Revised: 24 June 2021

Accepted: 31 August 2021

\section{Key words:}

Refugee mental health; refugee camps; conflict; displacement; health policy; barriers to care

Author for correspondence:

Eric Rafla-Yuan,

E-mail: eraflayuan@ucsd.edu (c) The Author(s), 2021. Published by Cambridge University Press. This is an Open Access article, distributed under the terms of the Creative Commons AttributionNonCommercial licence (http://creative commons.org/licenses/by-nc/4.0/), which permits non-commercial re-use, distribution, and reproduction in any medium, provided the original article is properly cited. The written permission of Cambridge University Press must be obtained prior to any commercial use.

\begin{abstract}
Background. Jordan has received more than three million refugees from bordering countries during times of conflict, including over 600000 Syrian refugees between 2011 and 2021 . Amidst this humanitarian crisis, a new mental health system for Syrian refugees has developed in Jordan, with most clinical services administered through non-governmental organizations. Prior studies have identified increased risk of psychiatric disorders in refugee populations and significant barriers for Syrian refugees seeking mental health treatment, but few have reviewed the organization or ability of local systems to meet the needs of this refugee population.

Methods. Qualitative interviews of mental health professionals working with refugees in Jordan were conducted and thematically analyzed to assess efficacy and organizational dynamics.

Results. Interviewees described barriers to care inherent in many refugee settings, including financial limitations, shortages of mental health professionals, disparate geographic accessibility, stigma, and limited or absent screening protocols. Additional barriers not previously described in Jordan were identified, including clinician burnout, organizational metrics restricting services, insufficient visibility of services, and security restrictions. Advantages of the Jordanian system were also identified, including a receptive sociopolitical response fostering coordination and collaboration, open-door policies for accessing care, the presence of community and grassroots approaches, and improvements to health care infrastructure benefiting the local populace.

Conclusions. These findings highlight opportunities and pitfalls for program development in Jordan and other middle- and low-income countries. Leveraging clinician input can promote health system efficacy and improve mental health outcomes for refugee patients.
\end{abstract}

\section{Introduction}

Armed conflict in Syria began in 2011, resulting in massive forced displacement of the Syrian population. As of May 2021, there were 666692 registered Syrian refugees residing in Jordan (UNHCR, 2021), a number that has been relatively stable since 2014 as the need far exceeds the rate of resettlement (UNHCR, 2021).

The mental health burden of refugees worldwide has been well documented (Bogic et al., 2015), with Syrian refugees commonly experiencing trauma from violence, the destruction of their homes and livelihood, loss of loved ones to death or separation, and extended periods in refugee camps. Syrian refugees in Jordan access mental health services via three distinct pathways: direct care at a mental health clinic, referral to specialty care by nongovernmental organizations (NGOs) or primary health professionals, or field-based outreach teams (Fig. 1. Within camps, the United Nations High Commissioner for Refugees (UNHCR) in partnership with foreign and domestic governments and NGOs provide shelter, food, education, and some healthcare services (Bloch and McKay, 2017).

There are distinctions between services available inside and outside camps, notable as approximately $80 \%$ of Syrian refugees in Jordan reside in urban areas, while the remaining live in refugee camps (UNHCR, 2021). Those outside of camps face many difficulties accessing healthcare services, with some studies finding 58-79\% of urban refugees unable to access physical or mental health care (UNHCR, 2015; Ay et al., 2016), despite UNHCR registration allowing Syrian refugees to access some services for free or at a subsidized cost (Akik et al., 2019).

Numerous prior studies have described barriers to mental health treatment for Syrian refugees in receiving countries, including financial limitations, stigmatization, distrust in healthcare systems, poor accessibility of services, misdiagnosis or lack of screening for mental health symptoms in primary settings, shortage of mental health professionals, communication and language barriers, and legal and immigration issues (Table 1). Although these general barriers to refugee mental health services have been well-documented, there has been minimal 


\section{Mental Health Service Accessibility for Syrian Refugees in Jordan}

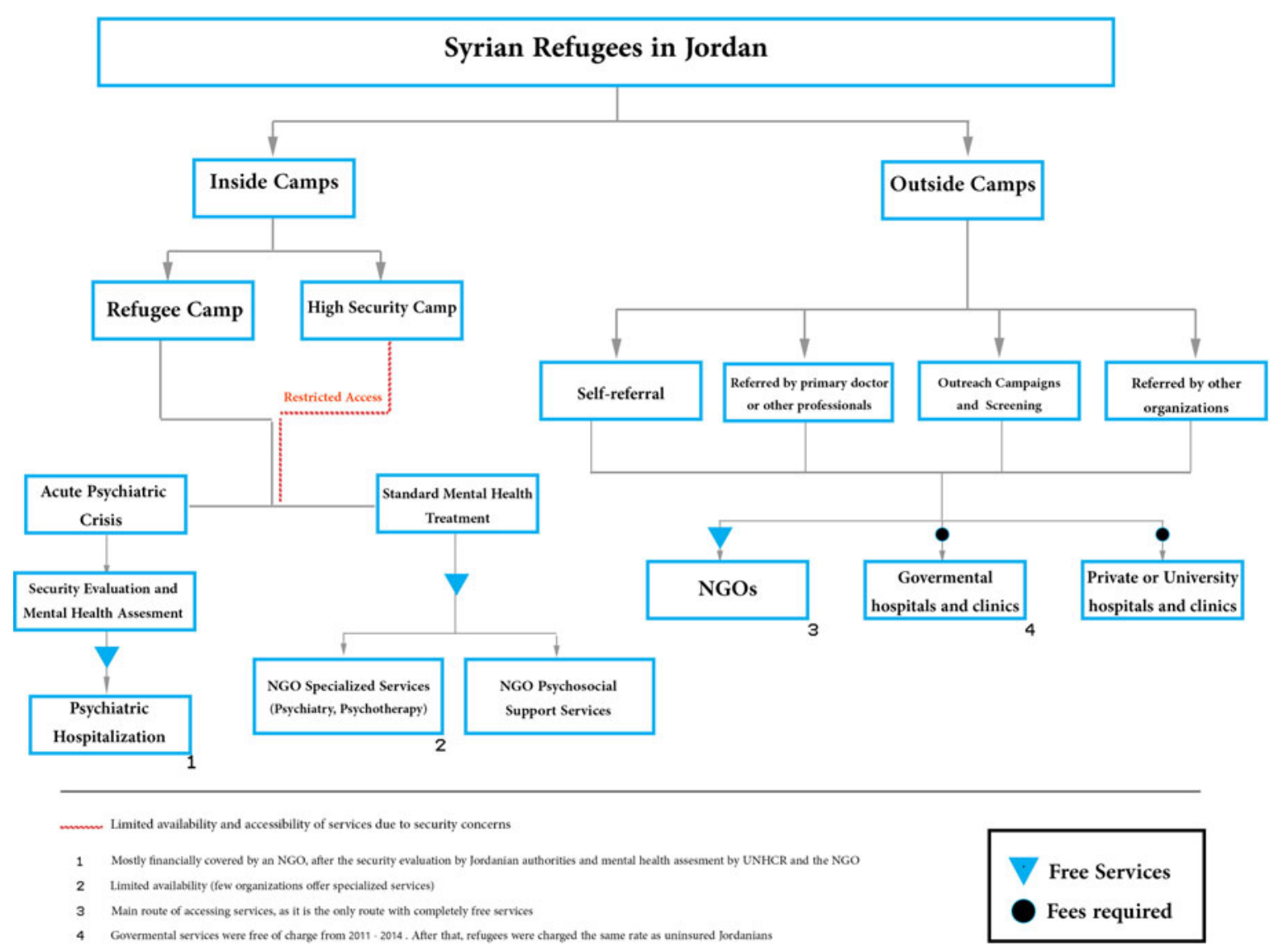

Fig. 1. Utilizing clinician input to improve refugee mental health.

investigation of their occurrences in Jordan, despite Jordan's key role in regional stability, brokering foreign humanitarian aid, and hosting refugees. Through numerous local and foreign stakeholders, a new mental health system for Syrian refugees has developed in Jordan, with most clinical services administered through NGOs (IMC, 2017). In this study, we seek to evaluate the strengths and weaknesses of this system and investigate how the current Jordanian model addresses these barriers (Table 1).

\section{Methods}

\section{Design}

An ethnographic approach was chosen to best capture the complex dynamics in which refugee health professionals practice. This method emphasizes how people understand their environment, and is ideal for acquiring a narrative account of a specific setting against a theoretical backdrop, such as the wide-scale changes of globalization and migration (Allard and Anderson, 2005).

\section{Interview structure and the interviewing process}

A semi-structured format was used, with initial structured questions covering multiple thematic sections: demographic information, clinical experience with refugee mental health, the environment of their system of care, organizational dynamics, and barriers to treatment. The unstructured portion of the interview allowed participants to expand on the initial topics or identify further areas of importance. Questions were formulated from literature review of barriers to care (summarized in Table 1) and pilot interviews were conducted to determine relevance and refine question structure. All participants were interviewed individually between November 2019 and March 2020. Interviews were conducted in Arabic, and interview length ranged between 60 and $123 \mathrm{~min}$ ( $\mathrm{mean}=78 \mathrm{~min})$. All interviews, transcription, and translation for analysis were completed by two of the study authors in tandem (M.A. and M.A.A), both fluent in English and Arabic and having multiple years of Arabic-English translation experience for scientific and popular media publications.

\section{Survey demographics}

The interview sample included 20 participants who reside in Jordan and provide psychiatric or psychosocial services to Syrian refugees. The sample included representation from different cities throughout Jordan, as well as professionals in various settings, including inside and outside refugee camps. Professional credentials of participants included psychiatrists, psychologists, counselors, social workers, mental health case managers, and psychosocial program officers and managers. All worked for local or international NGOs. (Appendix Table 1) 
Table 1. Barriers to mental health service for Syrian refugees in host countries

\begin{tabular}{|c|c|}
\hline Barrier & Description \\
\hline Financial limitations & $\begin{array}{l}\text { - Often a primary barrier to access } \\
\text { - Includes costs of initial consultation, continuing care, medication, and transportation } \\
\text { - Stem from limited funding pools for organizations and minimal financial means for most refugee } \\
\text { individuals and families } \\
\text { (Doocy et al., 2016; Al-Rousan et al., 2018; Dator et al., 2018; El-Arab and Sagbakken, 2018; } \\
\text { Doğan et al., 2019). }\end{array}$ \\
\hline Stigmatization & $\begin{array}{l}\text { - Significant barrier to mental health treatment in many other refugee-receiving countries } \\
\text { - Refugees may avoid care to due to stigma of psychiatric diagnoses or treatment } \\
\text { - Refugees may be especially cautious in avoiding further stigmatization as they attempt to } \\
\text { resettle in a new country } \\
\text { (Hassan et al., 2015; Ay et al., 2016; Sijbrandij et al., 2017; Silove et al., 2017). }\end{array}$ \\
\hline Distrust in the mental healthcare system & $\begin{array}{l}\text { - Many refugees have skeptical views of organizations and mental health professionals. } \\
\text { - Mistrust may be well-founded due to poor or traumatic prior experiences. } \\
\text { - Clinicians may be viewed as biased or unreliable due to the differing religious denomination, } \\
\text { ethnic affiliation, or political orientation. } \\
\text { - Unfamiliarity or lack of education on mental health diagnoses and treatment may also engender } \\
\text { distrust or confusion. } \\
\text { (Hassan et al., 2015; Majumder et al., 2015; Hebebrand et al., 2016; Sijbrandij et al., 2017; } \\
\text { Silove et al., 2017). }\end{array}$ \\
\hline Accessibility & $\begin{array}{l}\text { - Services in Jordan for refugees outside of camps are typically based in urban centers. } \\
\text { - Refugees in rural areas may be unable to access these services due to cost and accessibility of } \\
\text { transportation } \\
\text { - Limited awareness of availability or locations to access these services } \\
\text { (Ay et al., 2016; Sijbrandij et al., 2017; Silove et al., 2017; El-Arab and Sagbakken 2018; Doğan } \\
\text { et al., 2019). }\end{array}$ \\
\hline $\begin{array}{l}\text { Misdiagnosis and lack of screening for mental health } \\
\text { symptoms in primary settings }\end{array}$ & $\begin{array}{l}\text { - Some studies suggest that low rates of mental health symptom reporting can be attributed to } \\
\text { lack of screening protocols and poor recognition in primary care settings (Hassan et al., 2015; Ay } \\
\text { et al., 2016). } \\
\text { - Another reported example includes refugees requesting medical or neurological evaluation } \\
\text { when psychiatric evaluation is indicated (Hassan et al., 2015). } \\
\text { - Prior studies frequently recommend that primary healthcare professionals receive further } \\
\text { training to recognize, diagnose and refer psychiatric patients (Ay et al., 2016). }\end{array}$ \\
\hline Shortage in mental health professionals & $\begin{array}{l}\text { - Insufficient numbers of mental health care professionals are available to address the needs of } \\
\text { refugees in many host countries, including in Jordan (Ay et al., 2016; Doğan et al., 2019). } \\
\text { - High rates of organizational and staff turnover further contribute to shortages (Salemi et al., } \\
\text { 2018). }\end{array}$ \\
\hline Communication and language barriers & $\begin{array}{l}\text { - In non-Arabic speaking countries, communication issues pose significant difficulties (Sijbrandij } \\
\text { et al., 2017; Doğan et al., 2019). } \\
\text { - Refugees may be hesitant to utilize interpreters due to worries about confidentiality, } \\
\text { compromising the therapeutic alliance between the therapist and the patient (Sijbrandij et al., } \\
\text { 2017; Doğan et al., 2019). } \\
\text { - Previous studies suggest this may be an uncommon occurrence in Jordan, an Arabic speaking } \\
\text { country with a similar cultural and historical context to Syria (Nicholas Seeley 2012; Syria Direct } \\
\text { 2019). }\end{array}$ \\
\hline Legal and immigration issues & $\begin{array}{l}\text { - Registration documents typically allow refugees to receive health services for free or greatly } \\
\text { discounted rates. } \\
\text { - Lack of documentation may also restrict access to other important social determinants of } \\
\text { health, such as food and housing (International Organization of Migration 2021). } \\
\text { - Fear of being reported to authorities poses another barrier to treatment (El-Arab and Sagbakken } \\
\text { 2018; Doğan et al., 2019). }\end{array}$ \\
\hline
\end{tabular}

\section{Data analysis}

Analysis of interview transcriptions proceeded through thematic clustering and coding. Themes included those previously mentioned in the literature, as well as novel themes not previously identified, and were sorted into system barriers and strengths. All coding was done by the authors in tandem to ensure reliability. 


\section{Ethical considerations}

Ethical approval for this work was granted by the Institutional Review Board (IRB) of Jordan University of Science and Technology, Irbid, Jordan. Study participation was voluntary with no compensation provided, and all participants provided informed consent prior to study enrollment.

\section{Results}

\section{Barriers}

Financial barriers in the Jordanian context

Consistent with prior studies, financial challenges were identified as the most significant barrier to refugee services in Jordan. Clinicians reported service restrictions due to funding and lack of personnel and supplies. Medication shortages were a particular bottleneck, leading to prescriptions for less-preferred medication options or unavailability of psychotropics altogether.

In addition to organizational financial limitations, the financial hardship of refugee patients was also a significant limiting factor to accessing care. Clinicians reported that participation in treatment was often interrupted due to refugee patients having difficulty accessing, and thus prioritizing, basic needs. An additional distinct financial challenge for refugees residing outside of camps was cost and availability of transportation to treatment centers.

Burnout and shortages of qualified mental health professionals Delays in treatment, including evidence-based psychotherapeutic interventions and psychotropic medication also arose from a shortage of mental health clinicians, including psychiatrists, psychologists, social workers, and counselors. Recommendations for improvement included increasing access to training for highly utilized trauma therapy modalities. Increasing rates of burnout among colleagues was also identified as an important factor to address the shortage of clinicians. Some clinicians became emotional when discussing these topics, highlighting the need for further organizational support.

\section{Systemic and organizational barriers}

Numerous organizational issues were identified that impacted treatment, leading to adverse outcomes, clinician burnout, and poor project sustainability. Lack of organizational foresight and sustainability of projects and services was felt to pose challenges to long-term change. Disruptions in continuity or ability to access care were some of the reported consequences.

Interviewees reported that organizational focus on preconceived target metrics limited clinician effectiveness. These metrics often prioritized high patient volume over quality of services, and were inflexible regarding number of sessions or the patient's condition or severity. Some respondents noted programs' problematic reliance on these metrics to ensure continued financial support.

All surveyed professionals commented on the inefficiency of communication and coordination between organizations, exemplified by no system for sharing records between organizations, or for screening, reporting, and documenting services. This led to suboptimal outcomes, service delays, duplication of services, unreliable referral processes, and lack of accountability. Some commented that UNHCR had begun to create a system for documentation and coordination, but it was not yet operational.
Surveyed professionals uniformly advocated for better reporting, oversight, and follow up mechanisms.

The above organizational processes promoted burnout among clinicians, compounded by reducing time or availability of resilience-building practices. Surveyed professionals uniformly recommended increased attention and organizational commitment to policies and practices responsive to the needs and input of both patients and clinicians.

\section{Stigma}

Respondents reported that stigma remains a major barrier to mental health treatment for refugees in Jordan, presenting as the stigmatization of individuals by society, and at times, the patient's own stigma toward mental health treatment. Self-stigma was described as most problematic at the beginning of the therapeutic process and lessening with sessions and time. Comparatively, social stigma was considered to be a continuing problem, and respondents described various strategies designed to help overcome it, including educational campaigns, normalization and increased visibility of services, physically co-locating mental health services with other medical services, and avoiding stigmatizing language in naming.

Hiring refugees as peer support professionals, community liaisons, or as mental health clinicians, was described as a particularly effective approach to reducing stigma and improving access. This strategy aligns with prior research findings and recommendations for using local strengths and resources to develop grassroots approaches for population health (Saraceno et al., 2007).

\section{Awareness, education, and screening}

Poor awareness of available mental health services was common and particularly notable for refugees residing outside camps and in rural areas. Absence of screening and misdiagnosis of psychiatric symptoms in primary settings was also identified as problematic, with reported repeating patterns of worsened case severity after delayed mental health care. Respondents uniformly commented on the importance of implementing screening protocols and improving education for non-mental-health professionals to better recognize mental health symptoms and refer patients when necessary. Notably, some participants were not familiar with the concept of screening, but were in favor of it once an explanation was provided.

\section{Limited accessibility in high-security camps}

A small number of high-security camps have been implemented by the Jordanian government due to fears of security threats. Currently, approximately 20000 Syrian refugees are housed in high-security camp settings (New York Post, 2018; PBS News, 2020). Mobility both for NGOs and refugees is highly restricted in these settings, greatly limiting access to all mental health services.

\section{Strengths}

\section{Supportive political and societal response}

Clinicians repeatedly commended the effectiveness of the Jordanian government, backed by popular support, in responding to the refugee crisis, specifically for its facilitation and cooperation with NGOs to administer refugee services. Interviewees also described Jordanian societal perceptions of refugees as neighbors, rather than intruders, highlighting the shared historic, cultural, 
Table 2. Clinician recommendations in Jordan

\begin{tabular}{ll} 
Clinician recommendations in Jordan & \\
\hline $\begin{array}{ll}\text { Improve communication and data sharing between } & \text { - Adopting standardized procedures } \\
\text { organizations } & \text { - Sharing documentation and patient enrollment status to avoid duplicate or redundant } \\
& \text { services } \\
\text { - Methods for quality review and improvement } & \text { - Improved referral and screening pathways. }\end{array}$
\end{tabular}

Implement mental health screening

- Screening protocols are an evidence-based method to improve access to care in resource-limited settings

- Can be implemented in primary health care or other intake settings

Targeted education for clinicians on refugee mental health
- Trauma-focused approaches and therapy modalities

- Accessibility to supervision and consultation

- Collaboration with local and international academic institutions $\begin{array}{ll}\text { Preventing burnout } & \text { - Important for organizations and clinicians alike } \\ & \text { - Flexible program policies and improved clinician autonomy may decrease burnout. } \\ & \text { - Increased study on effective support protocols for clinicians experiencing burnout }\end{array}$

Geographic accessibility

- Increase geographic distribution for service locations

- Attend to availability and affordability of transportation to services

- Security zones further restrict access and should receive focused attention
- Incorporating refugees as peer support professionals improves outcomes and decreases stigma

- Open door policies improve access to care and religious values of the region. Nearly all clinicians reported that historic ties between Jordan and Syria, particularly in culture and language, promoted integration of refugees in mental health programs, and Jordanian society as a whole.

\section{Organizational open-door policies}

Offering services to all refugees was highlighted as an effective strategy in Jordan. Many interviewees reported that organizational policies offering services to refugees regardless of circumstances, legal status, or socioeconomic level, allowed them to practice in accordance with their personal and professional ethics. Furthermore, responses indicated lack of documentation was uncommon, and when it did occur, did not pose a barrier to provision of services.

\section{Effects on the Jordanian healthcare infrastructure}

This newly developing system of mental health services for refugees was reported to have regionally advanced the field of mental health and brought new economic and educational opportunities for Jordanian students and professionals. Many described personally learning new skills and finding more career advancement opportunities. This new system has also increased the breadth and availability of services offered to Jordanian citizens, as many NGOs have an external or internal mandate to concurrently provide services to Jordanians.

\section{Discussion}

The Jordanian response to the Syrian crisis has allowed for development of a new system of mental healthcare for refugees. In collaboration with the UNHCR and numerous NGOs, a wide spectrum of mental services are offered and continue to grow.
Significant barriers remain to improving accessibility of mental health services for Syrian refugees in Jordan, including financial limitations, transportation difficulties, clinician shortages and burnout, inflexible organizational policies, treatment stigma, limited or absent screening protocols, and security restrictions in high-security settings.

A positive sociopolitical response by Jordanian leadership, organizational open-door policies, and contributions to Jordan's healthcare infrastructure were described as major advantages of the Jordanian model.

Clinician input provides an effective mechanism to understand and improve this system (Table 2). Addressing these barriers and capitalizing on system strengths may improve access and outcomes for refugee patients in Jordan and other middle or low income settings worldwide.

\section{Study limitations and future directions}

Some clinicians reported siloing or partitioning of services and had difficulty commenting on larger systemic factors, thus our results may be limited by the clinical focus of study participants. These findings would be strengthened by input from patients and non-clinician stakeholders, including government officials and NGO administrators. Further quantitative investigation may also strengthen these findings and allow for additional analysis across multiple settings. The humanitarian crisis in Syria began in 2011 and continues to evolve-and with it changes the needs of displaced individuals. Continuous appraisal of this developing healthcare system should be undertaken.

Supplementary material. The supplementary material for this article can be found at https://doi.org/10.1017/gmh.2021.36. 
Acknowledgements. The authors would like to thank Dina Aqrabawi, Alaa Shehadeh, Rand Al-Soleiti and Dr. Ala'a Al-Froukh for their continued help and support through the time of this project. We are also indebted to all the interview participants who graciously shared their time and expertise.

Financial support. This research received no specific grant from any funding agency, commercial, or not-for-profit sectors.

Conflicts of interest. Drs. Rafla-Yuan, Al-Soleiti, and Nashwan have no conflicts of interest to disclose, financial or otherwise. Mr. Abu Adi is employed by CIVIC<www.civic.co $>$, an international aid NGO with operations in Jordan. Mr. Abu Adi and CIVIC are not involved in healthcare services and Mr. Abu Adi did not receive any incentives to participate in this research work, financial or otherwise.

\section{References}

Akik C, Ghattas H, Mesmar S, Rabkin M, El-Sadr WM and Fouad FM (2019) Host country responses to non-communicable diseases amongst Syrian refugees: a review. Conflict and Health 13, 8.

Al-Rousan T, Schwabkey Z, Jirmanus L and Nelson BD (2018) Health needs and priorities of Syrian refugees in camps and urban settings in Jordan. Eastern Mediterranean Health Journal 24, 243-253.

Allard F and Anderson E (2005) Ethnography. In Kempf-Leonard K (ed.), Encyclopedia of Social Measurement. Amsterdam: Elsevier, pp. 833-843.

Ay M, González PA and Delgado RC (2016) The perceived barriers of access to health care among a group of non-camp Syrian refugees in Jordan. International Journal of Health Services 46, 566-589.

Bloch A and McKay S (2017) Living on the margins: undocumented migrants in a global city. Journal of Law and Society 43, 469-473.

Bogic M, Njoku A and Priebe S (2015) Long-term mental health of warrefugees: a systematic literature review. BMC International Health and Human Rights 15, 29.

Dator W, Abunab H and Dao-Ayen N (2018) Health challenges and access to health care among Syrian refugees in Jordan: a review. Eastern Mediterranean Health Journal 24, 680-686.

Doğan N, Dikeç G and Uygun E (2019) Syrian refugees' experiences with mental health services in Turkey: "I felt lonely because I wasn't able to speak to anyone.". Perspectives in Psychiatric Care 55, 673-680.

Doocy S, Lyles E, Akhu-Zaheya L, Burton A and Burnham G (2016) Health service access and utilization among Syrian refugees in Jordan. International Journal for Equity in Health 15, 108.

El-Arab R and Sagbakken M (2018) Healthcare services for Syrian refugees in Jordan: a systematic review. European Journal of Public Health 28, 1079-1087.

Hassan G, Kirmayer LJ, MekkiBerrada A, Quosh C, el Chammay R, Deville-Stoetzel JB, Youssef A, Jefee-Bahloul H, Barkeel-Oteo A, Coutts A, Song S and Ventevogel P (2015) Culture, Context and the Mental Health and Psychosocial Wellbeing of Syrians: A Review for Mental Health and Psychosocial Support staff working with Syrians Affected by Armed Conflict. Geneva: UNHCR.

Hebebrand J, Anagnostopoulos D, Eliez S, Linse H, Pejovic-Milovancevic M and Klasen H (2016) A first assessment of the needs of young refugees arriving in Europe: what mental health professionals need to know. European Child and Adolescent Psychiatry 25, 1-6.
International Medical Corps (IMC) (2017) Who is Doing What Where and When in Mental Health and Psychosocial Support in Jordan? - 2017 Interventions Mapping Exercise. (https://reliefweb.int/report/jordan/whodoing-what-where-and-when-4ws-mental-health-psychosocial-support-jordan-2017). Accessed 7 February 2021.

International Organization of Migration (2021) Social Determinants of Migrant Health. (https://www.iom.int/social-determinants-migrant-health). Accessed 16 June 2021.

Majumder P, O'Reilly M, Karim K and Vostanis P (2015) 'This doctor, I not trust him, I'm not safe': the perceptions of mental health and services by unaccompanied refugee adolescents. International Journal of Social Psychiatry 61, 129-136.

New York Post (2018) Over 8000 Syrians still being held in Jordanian no-go camp, Associated Press. (https://nypost.com/2018/01/30/over-8000-syriansstill-being-held-in-jordanian-no-go-camp/). Accessed 7 February 2021.

Nicholas Seeley. Jordan's "open door" policy for Syrian refugees. Foreign Policy. (https://foreignpolicy.com/2012/03/01/jordans-open-door-policy-forsyrian-refugees/). Accessed 7 February 2021.

PBS News. Most aid to Syria's Rukban camp has been blocked for more than a year: Here's why. (https://www.pbs.org/newshour/world/most-aid-to-syriasrukban-camp-has-been-blocked-for-more-than-a-year-heres-why). Accessed 7 February 2021.

Salemi C, Bowman J and Compton J (2018) Services for Syrian Refugee Children and Youth in Jordan: Forced Displacement, Foreign Aid, and Vulnerability. Economic Research Forum Working Paper Series. (https://erf. org.eg/publications/services-for-syrian-refugee-children-and-youth-in-jordanforced-displacement-foreign-aid-and-vulnerability/). Accessed 7 February 2021.

Saraceno B, van Ommeren M, Batniji R, Cohen A, Gureje O, Mahoney J, Sridhar D and Underhill C (2007) Barriers to improvement of mental health services in low-income and middle-income countries. The Lancet 370, 1164-1174.

Sijbrandij M, Acarturk C, Bird M, Bryant RA, Burchert S, Carswell K, de Jong J, Dinesen C, Dawson KS, El Chammay R, van Ittersum $L$, Jordans M, Knaevelsrud C, McDaid D, Miller K, Morina N, Park AL, Roberts B, van Son Y, Sondorp E, Pfaltz MC, Ruttenberg L, Schick M, Schnyder U, van Ommeren M, Ventevogel P, Weissbecker I, Weitz E, Wiedemann N, Whitney C and Cuijpers P (2017) Strengthening mental health care systems for Syrian refugees in Europe and the Middle East: integrating scalable psychological interventions in eight countries. European Journal of Psychotraumatology 7, 8.

Silove D, Ventevogel P and Rees S (2017) The contemporary refugee crisis: an overview of mental health challenges. World Psychiatry 16, 130-139.

Syria Direct. 'No place for hate': Eight years later, Syrians still feel welcomed in Jordan. (https://syriadirect.org/news/\%E2\%80\%98no-place-for-hate\% E2\%80\%99-eight-years-later-syrians-still-feel-welcomed-in-jordan/). Accessed 7 February 2021.

United Nations High Commissioner for Refugees (UNHCR) (2015) At a glance: Health access and utilization survey among non-camp refugees in Jordan. (https://reliefweb.int/report/jordan/glance-health-access-and-utilization-survey-among-non-camp-refugees-jordan-may-2015). Accessed 23 June 2021.

United Nations High Commissioner for Refugees (UNHCR) - Syria Regional Response Inter-agency Information Sharing Portal. (https:// data2.unhcr.org/en/situations/syria/location/36). Accessed 23 June 2021. 